\title{
CIRUGÍA MÍNIMAMENTE INVASIVA: PROMONTOFIJACIÓN LAPAROSCÓPICA CON MAШA Y ANCLAJE DE TITANIO PARA PROLAPSO DE CÚPULA VAGINAL
}

\author{
Cristián Pomés $C .^{1}$, Rodrigo Pineda A. ${ }^{1}$, Sebastián Ramírez A. ${ }^{a}$ \\ ${ }^{1}$ Unidad de Ginecología, Departamento de Obstetricia y Ginecología, Facultad de Medicina, Pontificia Universidad \\ Católica de Chile.
}

Interno, Escuela de Medicina, Pontificia Universidad Católica de Chile.

\section{RESUMEN}

Objetivo: Analizar y evaluar la factibilidad técnica, eficacia y seguridad de la promontofijación laparoscópica con malla y anclajes helicoidales de titanio para el tratamiento del prolapso de cúpula vaginal. Método: Entre noviembre de 2005 y julio de 2006, en el Servicio de Ginecología del Hospital Clínico de la Universidad Católica, se efectuaron 5 promontofijaciones laparoscópicas, utilizando técnica consistente en malla unida al promontorio del sacro con anclajes helicoidales de titanio. El rango de edad de las pacientes fue entre 46 y 65 años, con paridad vaginal entre 2 y 3 . Resultados: La técnica quirúrgica logró corregir el defecto en las 5 pacientes. No hubo complicaciones intraoperatorias, ni post operatorias, con rápida recuperación y reintegración a las actividades habituales. Conclusiones: Nos enfrentamos a una novedosa técnica, segura y reproducible, con las ventajas de la laparoscopia, alta precoz y estéticamente óptima.

PALABRAS CLAVES: Promontofijación laparoscópica, mallas, anclajes helicoidales de titanio

\section{SUMMARY}

Objective: To analyze and evaluate the technical feasibility, effectiveness and security of the laparoscopic sacrocolpopexy with mesh and helical titanium anchorages for the treatment of vaginal vault prolapse. Method: Between November 2005 and July 2006, five laparoscopic sacrocolpopexies, using this technique, took place in the Gynecology Service of the Clinical Hospital of Pontificia Universidad Católica de Chile, consisting of mesh joined to the sacro promontory with helical titanium anchorages. The age of the patients was between 46 and 65 years, with vaginal parity between 2 and 3 . Results: In all patients the surgical technique corrects the defect. There were no interoperating or postoperating complications, with a prompt recovery and reintegration to usual activities. Conclusions: We face a novel, safe and reproducible technique, with the advantages of the laparoscopy, early leave hospital and esthetically optimum.

KEY WORDS: Laparoscopic sacrocolpopexy, mesh, helical titanium anchorages

\section{INTRODUCCIÓN}

El prolapso de cúpula vaginal es una complicación producto del debilitamiento de la fascia endopélvica en el área del vértice vaginal, y del complejo cardinal-úterosacro (1), descrito por De Lancey en 1992, quien en relación a la anatomía del piso pelviano definió 3 niveles de sustentación útero-vaginal; el primero constituido por el complejo ligamentoso cardinal-úterosacro, el segundo por 
Tabla I

RESUMEN DE PACIENTES SOMETIDAS A PROMONTOFIJACIÓN LAPAROSCÓPICA CON MALLA Y ANCLAJES HELICOIDALES DE TITANIO

\begin{tabular}{|c|c|c|c|c|c|c|c|}
\hline Caso & Edad & Paridad & Antecedentes & $\begin{array}{l}\text { Grado col- } \\
\text { pocele }\end{array}$ & $\begin{array}{l}\text { Prolapso } \\
\text { asociado }\end{array}$ & $\begin{array}{l}\text { Cirugía com- } \\
\text { plementaria }\end{array}$ & $\begin{array}{l}\text { Tiempo qui- } \\
\text { rúrgico (min) }\end{array}$ \\
\hline 1 & 65 & 3 PVE & $\begin{array}{l}\text { HT con SOB, } \\
\text { vía abdominal }\end{array}$ & IV reductible & $\begin{array}{l}\text { Cistocele grado II. } \\
\text { Sin rectocele }\end{array}$ & - & 125 \\
\hline 2 & 47 & 3 PVE & $\begin{array}{l}\text { HT, vía vaginal } \\
\text { asistida por } \\
\text { laparoscopía }\end{array}$ & III reductible & $\begin{array}{l}\text { Cistocele moderado. } \\
\text { Rectocele. }\end{array}$ & $\begin{array}{l}\text { Hemicolectomía por } \\
\text { dólicomegacolon }\end{array}$ & 240 \\
\hline 3 & 56 & 3 PVE & $\begin{array}{l}\text { HT con SOB, } \\
\text { vía abdominal }\end{array}$ & I reductible & Cistocele severo & - & 170 \\
\hline 4 & 56 & $2 \mathrm{PVE}$ & HT, vía abdominal & III reductible & - & TVT-O por IOE & 210 \\
\hline 5 & 46 & $\begin{array}{l}3 \text { PVE } \\
1 \text { Cesárea }\end{array}$ & $\begin{array}{l}\text { HT, vía vaginal } \\
\text { a }\end{array}$ & I reductible & - & - & 120 \\
\hline
\end{tabular}

PVE: Partos vaginales espontáneos. HT: Histerectomía. SOB: Salpingooforectomía bilateral.

la fascia recto-vaginal y el tercero por el cuerpo perineal. Este primer nivel según De Lancey sería de importancia en la etiología del prolapso de cúpula (2).

Esta complicación puede ocurrir entre el 0,2 y $2 \%$ de las pacientes con el antecedente de histerectomía $(3,4)$, algunas series han publicado cifras desde un $2 \%$ hasta un $45 \%$ (1). Dado que la histerectomía es una cirugía frecuente, nos veremos enfrentados a diario en nuestra práctica clínica a pacientes que cursen con prolapso de cúpula vaginal.

Este defecto en ocasiones puede asociarse indirectamente a compromiso de intestino delgado, resultando en un contacto directo entre intestino con mucosa vaginal, conocido como enterocele (5).

Intentar solucionar el problema del prolapso de cúpula vaginal durante décadas ha sido un desafío. Lo que primero se describió en la literatura para el tratamiento del prolapso de cúpula vaginal, data de fines del siglo XIX y consistía en suspensión de la cúpula con fijación sacrotuberosa (4). A lo largo de los años, se han utilizado diferentes zonas o reparos anatómicos para la fijación de la cúpula: como los ligamentos sacroespinoso, ileococcigeo, úterosacro y finalmente el promontorio del sacro, como presentaremos en nuestro trabajo.

Debido a la variedad de alternativas, es que las técnicas quirúrgicas han incluido vías de abordaje como vaginal, abdominal, combinadas, y laparoscópica en los últimos años.
Por vía vaginal las técnicas más utilizadas son la colpopexia a ligamentos sacro espinosos o iliococcigeos y la culdoplastía high McCall. Sin embargo, en los casos sin actividad sexual, puede considerarse sólo la eliminación del prolapso sin

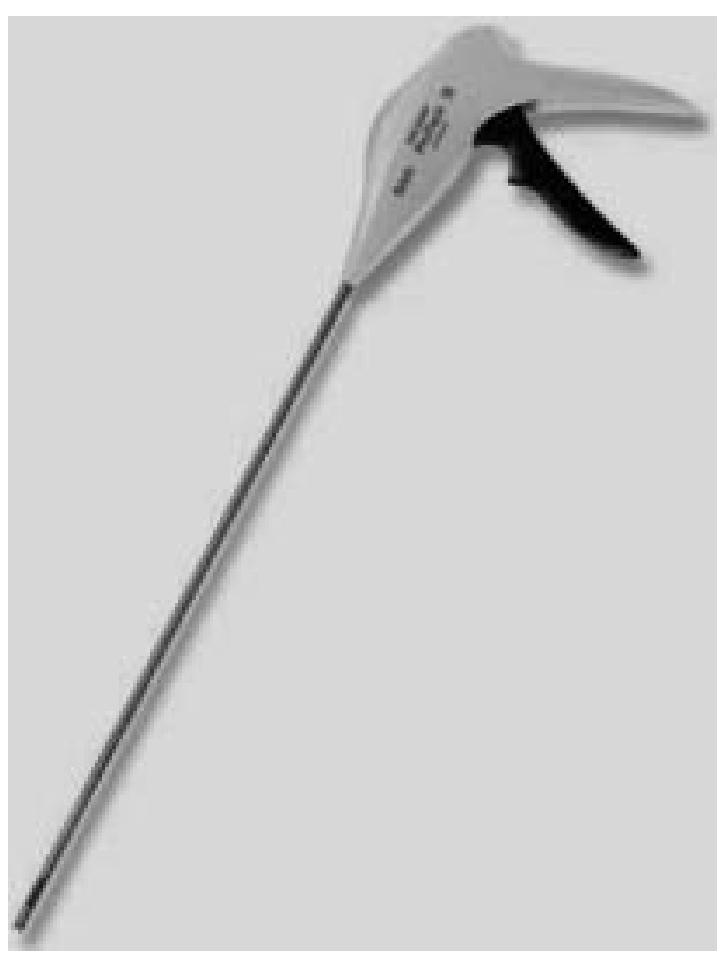

Figura 1. Autosutura de AutoSuture, ProTack, de $5 \mathrm{~mm}$ (Tyco). 


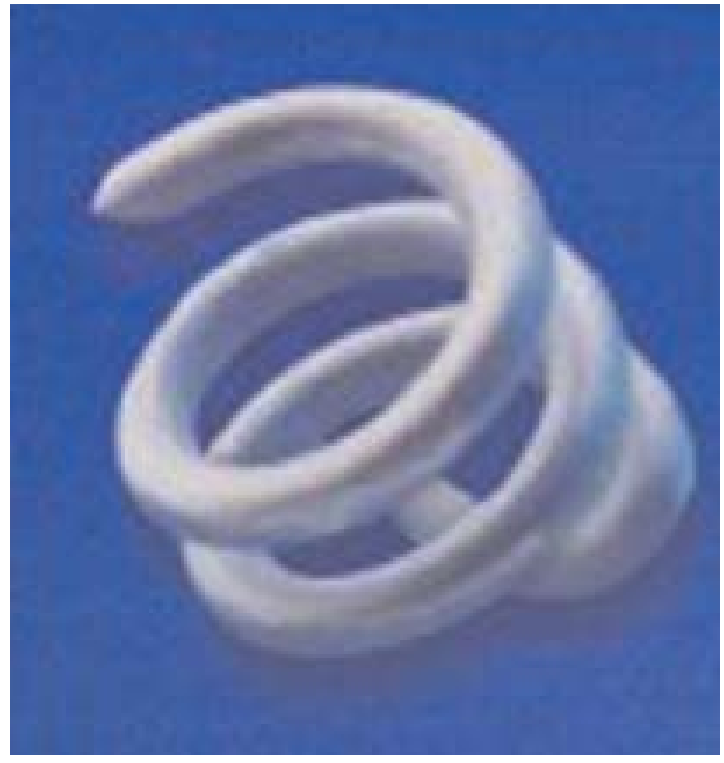

Figura 2. Anclajes helicoidales de titanio de $4 \mathrm{~mm}$, AutoSuture, ProTack, (Tyco).

preservar la función vaginal, en este tipo de pacientes se puede realizar colpocleisis (6).

En lo que respecta a la utilización de laparoscopia en el prolapso de cúpula vaginal están descritas dos técnicas: 1) Suspensión de la cúpula vaginal a los ligamentos útero sacros: disección de las fascias pubocervical y rectovaginal, reaproximación de ambas fascias, creándose una nueva cúpula vaginal que luego se suspende a los ligamentos úterosacros. 2) Sacro-colpofijación: utilizando malla que se sutura a la cúpula vaginal y al promontorio (7).

En nuestro país, las publicaciones en relación al tratamiento del prolapso de cúpula vaginal son variadas. Silva y cols (6) en 2004, presentaron una serie de casos tratados quirúrgicamente con técnica de IVS posterior (Posterior Intravaginal Slingplasty), también llamada sacropexia infracoccígea, con resultados satisfactorios aprovechando las ventajas de la cirugía mínimamente invasiva. Quilaqueo (8), presentó en 2005 su experiencia de 7 pacientes sometidas a corrección de prolapso genital completo por vía laparoscópica, con buenos resultados aprovechando las ventajas ya descritas que posee la cirugía laparoscópica, ventajas que nos interesa mostrar en nuestro estudio. Recientemente se presentó la experiencia de promontosuspensión con malla y anclajes helicoidales de titanio a través de minilaparotomía con separador Mobius ${ }^{\circledR}(9)$, técnica similar a la

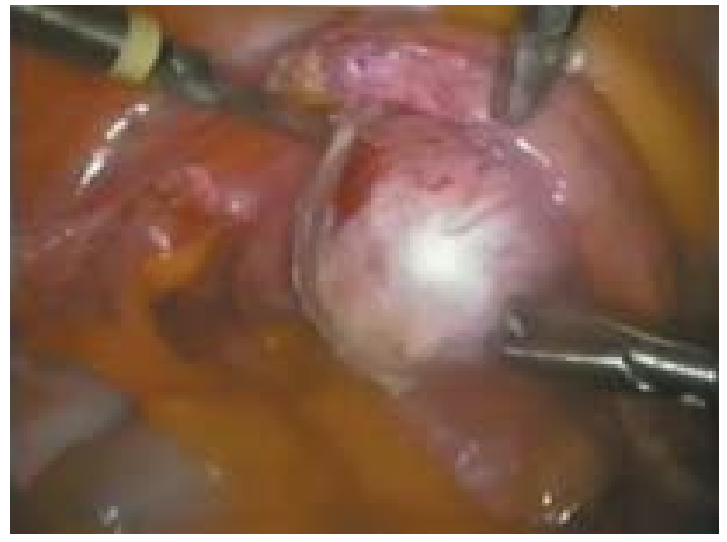

Figura 3. Disección de la cúpula.

que proponemos, sin embargo, nuestra diferencia radica en el abordaje vía laparoscopia.

En nuestra técnica se utiliza una malla de Vypro $I^{\circledR}$, en forma de pantalón como letra Y. Esta se sutura con puntos de prolene a la cúpula vaginal por cara posterior y anterior, para luego unir el brazo libre de la malla al sacro, mediante autosutura con anclajes helicoidales de titanio ProTack ${ }^{\circledR}$, que tienen la ventaja de ser hemostáticos y de disminuir la incidencia de periostitis. De hecho existen casos publicados de discitis luego de cirugía de prolapso de cúpula vía laparoscópica, rara pero importante complicación (10).

La autosutura de Auto Suture ${ }^{\circledR}$ ProTack $^{\circledR}$ de 5 $\mathrm{mm}$, permite una fácil maniobrabilidad con un largo de $35,5 \mathrm{~cm}$. Contiene 30 anclajes de titanio. El diámetro aproximado de los anclajes helicoidales es de $4 \mathrm{~mm}$ y un largo de $3,8 \mathrm{~mm}$. Sin embargo, la principal diferencia de nuestra técnica radica en

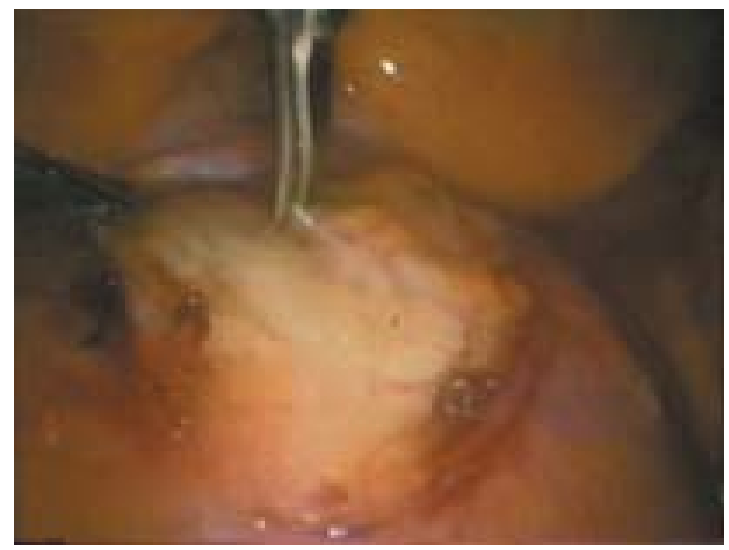

Figura 4. Disección del promontorio. 


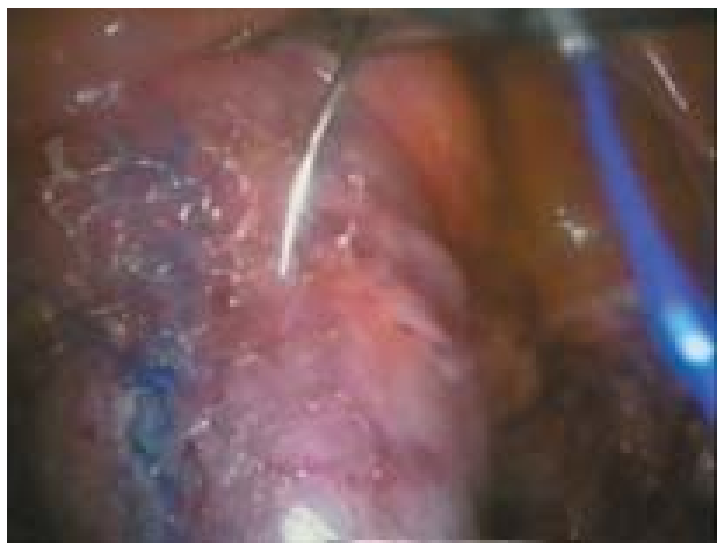

Figura 5. Fijación de la malla a la cara anterior de la cúpula.

que el abordaje es laparoscópico. Lo que entrega una serie de ventajas por sobre la cirugía abierta como son:

- visión superior para el cirujano gracias a la distensión de la pelvis por el neumoperitoneo.

- acceso menos traumático.

- menor utilización de analgésicos y mejor manejo del dolor.

- menos días de hospitalización y deambulación precoz.

- retorno precoz a las actividades habituales.

- disminución de los costos.

\section{PACIENTES Y MÉTODO}

En el Hospital Clínico de la Pontificia Universidad Católica de Chile, entre los meses de noviembre de 2005 y Julio de 2006, se realizó en cinco

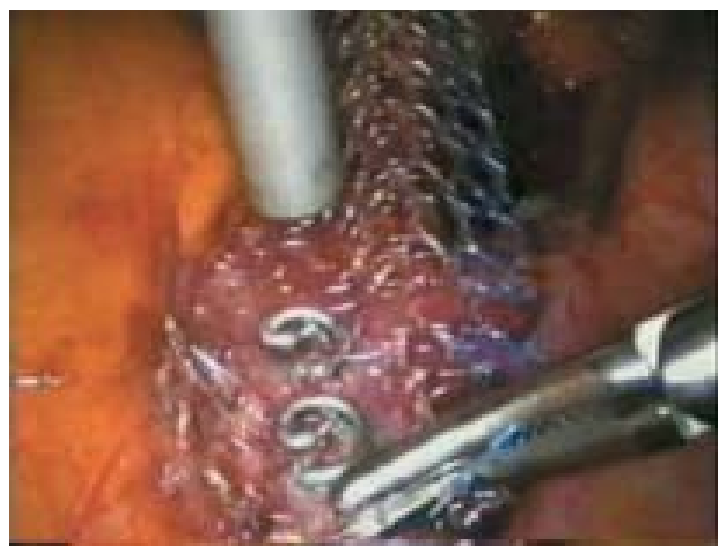

Figura 6. Anclaje de la malla al promontorio con sistema Protack ${ }^{\circledR}$. pacientes la corrección del prolapso de la cúpula vaginal mediante promontofijación laparoscópica. En cuatro casos se utilizó malla de Vypro $\|^{\circledR}$ y en un caso Gynemesh ${ }^{\circledR}$. Para la fijación de la malla al promontorio del sacro se utilizaron anclajes helicoidales de titanio ProTack ${ }^{\circledR}$.

Caso 1: Paciente de 65 años, multípara de 3 partos vaginales espontáneos. Antecedente de histerectomía total con salpingooforectomía bilateral, vía abdominal a los 50 años por miomas uterinos. Actualmente, colpocele severo (grado IV) y cistocele grado II. No se evidencia rectocele.

Caso 2: Paciente de 47 años, multípara de 3 partos vaginales espontáneos. Antecedente de histerectomía total vía vaginal asistida por laparoscopia, más plastia anterior y posterior por prolapso genital completo, en otro centro, a los 41 años. Actualmente, colpocele grado III, cistocele moderado y rectocele. Nuestra cirugía además fue complementada por equipo de coloproctología realizando una sigmoidectomía laparoscópica por dólicomegacolon.

Caso 3: Paciente de 56 años, multipara de 3 partos vaginales espontáneos. Antecedente de histerectomía total con salpingooforectomía bilateral, vía abdominal por patología ovárica a los 40 años. Actualmente, cistocele severo y colpocele grado I.

Caso 4: Paciente de 56 años, multípra de 2 partos vaginales espontáneos. Antecedente de histerectomía total vía abdominal por miomas uterinos. Actualmente, portadora de incontinencia de orina de esfuerzo (IOE) y colpocele grado III. Cistomanometría no presentó contracciones no inhibidas, así, la promontofijación se complementó con TVT-O.

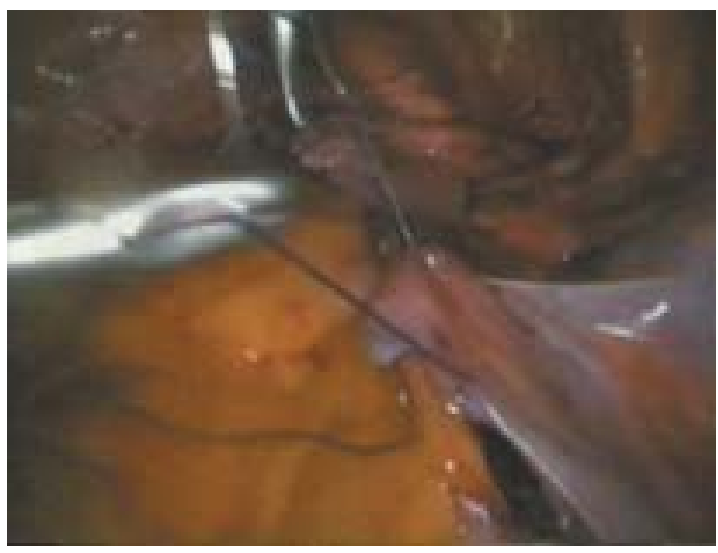

Figura 7. Peritonización de la malla. 
Caso 5: Paciente de 46 años, multípara de 4, 3 PVE y 1 cesárea. Con antecedente de histerectomía vaginal, a los 45 años por miomas uterinos. Actualmente, colpocele grado I.

Todas las pacientes fueron sometidas al procedimiento quirúrgico bajo anestesia general, previa preparación de colon. Recibieron cefazolina $1 \mathrm{~g} \mathrm{ev}$, una hora preoperatorio, luego $1 \mathrm{~g}$ ev cada ocho horas postcirugía. Se mantuvieron con sonda Foley las primeras veinticuatro horas. La analgesia se manejó con bomba de infusión continua: ketorolaco $90 \mathrm{mg}$ más tramadol $300 \mathrm{mg}$ en 250 cc, solución fisiológica $0,9 \%$ a $10 \mathrm{cc} /$ hora, durante las primeras 15 horas en promedio. Posteriormente ketorolaco $30 \mathrm{mg}$ ev cada ocho horas y morfina $3 \mathrm{mg}$, en caso de dolor. A partir de las 24 horas post cirugía se inició analgesia oral. Una vez iniciado el régimen hídrico se dejó profilaxis antifúngica, para malla Vypro $\|^{\circledR} \circ$ Gynemesh $^{\circledR}$, con fluconazol $150 \mathrm{mg}$ v.o. por una vez.

Técnica quirúrgica laparoscópica:

1. Posición de litotomía baja, aseptización, instalación de sonda foley.

2. Realización de neumoperitoneo con aguja de Verres umbilical, o por punto de Palmer (si existiera antecedente de cirugía previa y se sospechara adherencias). Se utilizó trocar central de $10 \mathrm{~mm}$ y tres punciones auxiliares de $5 \mathrm{~mm}$. En el caso 2, una de las punciones auxiliares fue de 10 $\mathrm{mm}$, para cirugía en segunda etapa por equipo de coloproctología.

3. Se exploró cavidad peritoneal y en todos los casos se constató ausencia de útero de acuerdo al antecedente de histerectomía.

4. Se disecó y liberó peritoneo que recubre domo de cúpula, se fijó malla en forma de pantalón (brazos cortos), con 3-4 puntos de prolene 3.0 a cúpula por cara posterior, y luego por anterior con 3-4 puntos del mismo material.

5. Se procedió a disecar el peritoneo parietal desde el promontorio del sacro hasta la pelvis menor, identificando previamente el ureter.

6. Se fijó malla en pantalón, extremo largo de la "Y" al promontorio del sacro con 3 suturas mecánicas Tyco en promedio.

7. Se peritonizó y cubrió con malla suturando con Vicry ${ }^{\circledR}$ 3-0.

8. Se procedió a revisar hemostasia y se cerró piel con monocril 3.0.

\section{RESULTADOS}

El tiempo quirúrgico considerando las cirugías complementarias, varió desde los 125 minutos hasta los 240 minutos en la cirugía más compleja. No hubo complicaciones intraoperatorias, ni postoperatorias de ningún tipo. El volumen de sangrado promedio no superó los 20-30 cc. Ninguno de los cinco casos requirió analgesia adicional. La realimentación se inició a las 6 horas de postoperadas con buena tolerancia, en el caso 2 se postergó hasta 48 horas por cirugía coloproctológica en segundo tiempo. Deambulación entre las 6 y 12 horas postcirugía, el alta ginecológica se dio a las 36 horas. En control a los 30 días del postoperatorio no se evidenciaron molestias ni complicaciones, se planificó control en 6 meses.

\section{DISCUSIÓN}

Este es el primer trabajo publicado en nuestro medio en relación a la promontofijación laparoscópica con malla y anclajes helicoidales de titanio. Nuestra breve experiencia, aún no nos permite hacer afirmaciones sobre la eficacia u otra característica de esta técnica, ni menos sugerirla.

La literatura en relación a la cirugía laparoscópica para el tratamiento del prolapso de cúpula vaginal, señala que la promontofijación tiene un mayor éxito a largo plazo que la suspensión de cúpula a los ligamentos uterosacros llegando a un índice de curación de un 92\% (7).

En relación a las ventajas de laparoscopía sobre laparotomía, un meta-análisis de la base Cochrane (11), identificó ocho estudios elegibles (Burton, 1997; Carey, 2000; Fatthy, 2001; Persson ,2000; Ross, 1995; Su, 1997; Summitt, 2000; Wallwiener, 1995). Cinco incluyeron 233 mujeres que recibieron laparoscopía y 254 colposuspensión abierta. Mientras por una parte la impresión subjetiva de cura de las mujeres pareció similar para ambos procedimientos hasta los 18 meses, hubo cierta evidencia de peores resultados para los procedimientos vía laparoscópica, en los resultados objetivos. Un único ensayo sugirió peor rendimiento a largo plazo, pero esto puede reflejar la inexperiencia quirúrgica de la colposuspensión laparoscópica (11).

En 2001, Carter y cols (12), publicaron una serie de 8 pacientes que fueron sometidos a suspensión de cúpula vaginal por vía laparoscópica, con una tasa de éxito de $100 \%$, a los 6 meses. Dos años más tarde, Serman y cols (13), reportaron una tasa objetiva de éxito de $100 \%$, con un seguimiento de 12 meses a 10 pacientes seleccionados con defectos en compartimento apical. El éxito total en una serie de 73 pacientes, alcanzó el $90 \%$ con seguimiento de 2 años de duración. 
Un tercer grupo, Nezhat y cols (14), publicaron una serie de 15 pacientes con éxito de $100 \%$, con un seguimiento entre 3-40 meses. Ross (15), presentó un $100 \%$ de éxito en 13 pacientes en un año de seguimiento. En 2004 el Departamento de Urología de la Universidad de Indianápolis, mostró una serie de 10 casos con seguimiento de 16 meses promedio, con buenos resultados y recomendación de la sacrocolpopexia laparoscópica para el tratamiento de prolapso de cúpula vaginal (16).

Sin embargo sólo estudios recientes están incluyen dentro de sus outcomes la satisfacción del paciente, de hecho una revisión desde el año 1972 al 2002 que incluyó 149 estudios con más de 6000 pacientes operados de prolapso por distintas vías, incluida la laparoscópica, evaluó los resultados que no mostraron diferencias en relación a tasa de curación y/o complicación. No existe estandarización en lo que a satisfacción respecta, de hecho es un outcome entregado por el paciente donde la tasa de curación es probablemente influenciada por el resultado funcional en términos de micción, defecación y actividad sexual, más que por el resultado anatómico obtenido (17).

Considerando lo anterior, creemos que esta técnica es una excelente alternativa para tratar quirúrgicamente el prolapso de cúpula vaginal posthisterectomía; con claras ventajas ya expuestas por sobre la laparotomía. Además, por vía laparoscópica no hay incisión vaginal, por ende nos entrega corrección, sin generar un tejido fibrótico, que posteriormente pudiera intervenir en un normal funcionamiento vaginal.

\section{CONCLUSIONES}

La promontofijación laparoscópica es una técnica reciente, mínimamente invasiva, con las ventajas propias de la laparoscopia, reproducible, efectiva y segura. Con una rápida recuperación y estéticamente óptima. Sin el riesgo de producir fibrosis a nivel vaginal y consecuente discomfort para la paciente, como podría ocurrir en cirugía por vía vaginal. Sin embargo, no existen estudios con seguimiento a largo plazo que nos permitan asegurar resultados a nuestros pacientes.

\section{BIBLIOGRAFÍA}

1. Flynn BJ, Webster GD. Surgical management of the apical vaginal defect. Curr Opin Urol 2002;12:353-8.

2. De Lancey JOL. Anatomic aspects of vaginal eversion after hysterectomy. Am J Obstet Gynecol 1992;166:1717-28.
3. Lemus SR, Martínez OA, Matute M, Sánchez A, Ramírez R, Hernández M. Sacropexy with abdominal fascia in treatment of vaginal prolapse vault of menopausal women. Ginecol Obstet Mex 2003;71 638-45.

4. Morley GW, Delancey JO. Sacroespinous ligament fixation for eversion of the vagina. Am J Obstet Gynecol 1998;158(4):872-81.

5. Richardson AC. The rectovaginal septum revisited: its relationship to rectocele and its importance in rectocele repair. Clin Obstet Gynecol 1993;36: 976-83.

6. Silva A, Ferrada L, Arroyo C, Nalbandian K. IVS (Posterior Intravaginal Slingplasty) para el tratamiento del prolapso de cúpula vaginal o procidencia de útero: serie de casos. Rev Chil OBstet Ginecol 2004;69(6):419-23.

7. Wattiez A, Mashiach R, Donoso M. Laparoscopic repair of vaginal vault prolapse. Curr Opin Obstet Gynecol 2003;15(4):315-9.

8. Quilaqueo M. Reparación del piso pélvico en prolapso genital completo vía laparoscópica. REV CHIL OBStet GINeCOL 2005;70(4):225-30.

9. Solà V, Pardo J, Ricci P, Guillof E, Alcalde J, Tacla X. Cirugía mínimamente invasiva para el tratamiento del prolapso de cúpula vaginal: promontosuspensión con malla y anclaje helicoidal de titanio, a través de minilaparotomía con separador elástico de automantención. Rev ChIL OBstet GineCOL 2005; 70(6):364-8.

10. Kapoor B, Toms A, Hooper P, Fraser AM, Cox CW. Infective lumbar discitis following laparascopic sacrocolpopexy. J R Coll Surg Edinb 2002;47(5): 709-10.

11. Moehrer B, Ellis G, Carey M, Wilson PD. Colposuspensión laparoscópica para la incontinencia urinaria en mujeres (Revisión Cochrane traducida). En: La Biblioteca Cochrane Plus 2006; Número 1.

12. Carter JE, Winter M, Mendehlsohn S, et al. Vaginal vault suspension and enterocele repair by Richardson-Saye laparoscopic technique; description of training technique and results. J Soc Laproend Surg 2001;5:29-36.

13. Serman El, Cook JR, O'Shea RT. Two-year experience with laparoscopic pelvic floor repair. J Am Assoc Gynecol Laparosc 2003;10:38-45.

14. Nezhat CH, Nezhat F, Nezhat C. Laparoscopic sacral colpopexy for vaginal vault prolapse. Obstet Gynecol 1994;4:381-3.

15. Ross JW. Apical vault repair, the cornerstone of pelvic vault reconstruction. Int Urogynecol J 1997; 8:146-52.

16. Sundaram CP, Venkatesh R, Landman J, Klutke CG. Laparoscopic sacrocolpopexy for the correction of vaginal vault prolapse. J Endourol 2004;18(7): 620-3.

17. Beer N, Kuhn A. Surgical techniques for vault prolapse: a review of the literature. Eur J Obstet Gynecol Reprod Biol 2005;119(2):144-55. 